\title{
Automated mapping of local layer slope and tracing of internal layers in radio echograms
}

\author{
Christian PANTON \\ Centre for Ice and Climate, Niels Bohr Institute, University of Copenhagen, Copenhagen, Denmark \\ E-mail: panton@nbi.ku.dk
}

\begin{abstract}
An automated method is presented for tracing layers in radio echograms. The method is designed to work with most radio-echo sounding echograms and has been successfully tested with a 180-210 MHz multichannel coherent depth sounder. To accurately trace layers, first approximate layer positions are calculated by integrating the local layer slope which is inferred by the intensity response to a slanted filter, then the positions are refined using an iterative optimization. The layers are traced using an active contour model or snake, which can be constrained to conserve both echogram features and smooth layers. With this technique it is possible to trace internal layers over distances of several hundred kilometers. The method was tested between two Greenland deep ice cores where the age-depth relation is known.
\end{abstract}

KEYWORDS: radio-echo sounding

\section{INTRODUCTION}

Radio-echo sounding (RES) is used to measure ice thickness and basal topography of ice sheets and glaciers, with measurements dating back to 1960, and internal stratigraphy in the echograms has been seen for nearly as long (Robin and others, 1969; Gudmandsen, 1975). The internal reflecting horizons (IRHs) are generally accepted to be isochrones (Siegert 1999; Hempel and others, 2000) and can, in some parts of the ice sheet, span several hundred kilometers in Greenland and several thousand kilometers in Antartica (Steinhage and others, 2013). In this paper, we refer to the IRHs as internal layers. Ice cores from the Greenland ice sheet are an archive of past climate, dating back up to 128.5 ka (NEEM Community Members, 2013); however, these cores represent point measurements on the vast ice sheet. The internal layers can be cross-linked with the highresolution ice-core record to provide a better understanding of the internal ice-sheet dynamics and changes to mass balance (Welch and Jacobel, 2003). The internal layers vary in depth and intensity; sometimes they even disappear and then reappear. The depth variability is caused by changes in accumulation (Baldwin and others, 2003), ice flow (Vaughan and others, 1999) and bedrock melt (Fahnestock and others, 2001), and the layer depth conversion from the two-way travel time of the radar wave is influenced by the firn density profile (Kovacs and others, 1995). Intensity variations caused by the limitations of the radar, including focusing and slope effects, have been described by Harrison (1971) and Jacobel and Hodge (1995). Other intensity variations are caused by physical features, such as attenuation from surface melt (Forster and others, 2014) and crevasses (Gades and others, 2000), or events during the acquisition, such as plane turns.

Manually picking internal layers is very time-consuming. Sime and others (2011) estimated that tracing 20 layers in a $20000 \mathrm{~km}$ RES survey would require 10 operator-years to complete. To reduce the manual workload, both commercially available software and custom-developed methods have been used to trace layers.

Commercial software originally developed for processing seismic datasets has been re-purposed into tracing RES layers: Landmark OpenWorks (used by, e.g., Eisen and others, 2004; Rotschky and others, 2004) and ReflexW by Sandmeier Scientific (used by, e.g., Karlsson and others, 2009; Mottram and others, 2009). The underlying tracking algorithms are not published, but the ReflexW 4.0 user manual says that the layer tracing is based on phase tracking and peak-following, and Eisen and others (2004) describe the operation of OpenWorks also as peak-following. Commercial software seems adequate to trace layers in specific studies, but the solutions leave no room for improvement or analysis of the tracing algorithms. The software also locks access to the algorithm such that specific format conversions might be needed to incorporate it into a processing workflow.

A number of tailored methods have been developed to extract features from RES echograms: Crandall and others (2012) used probabilistic scene labeling to detect the air-ice and ice-bedrock interface of echograms, Karlsson and others (2013) fitted a ramp function to detect a climatic transition, and Fahnestock and others (2001) and Baldwin and others (2003) traced layers using iterative peakfollowing and pattern matching.

One of the key challenges in constructing an automated tracking method for RES is the fact that in some regions the layers are closely spaced. This creates many identical, slightly offset, layer candidates, and if the algorithm chooses the wrong candidate, then it could divert into the neighboring layers.

Most of the layer-tracking methods referenced above use a peak-following scheme, which makes them liable to steer into neighboring layers in regions where the visible stratigraphy deteriorates. Instead of propagating the traced layer along the track, as these schemes do, a method that traces the layer by optimizing the position of the entire layer is preferable, as that could bridge areas with poor echogram quality.

The estimated slope of the layers can be used to form synthetic isochrones. ReflexW uses the phase of the reflected wave to infer the slope and steer the layer trace. One method for deriving layer slope from RES echogram intensity has been presented by Sime and others (2011), a multi-step procedure where the layer slope is inferred from measurements on segmented layer objects. 


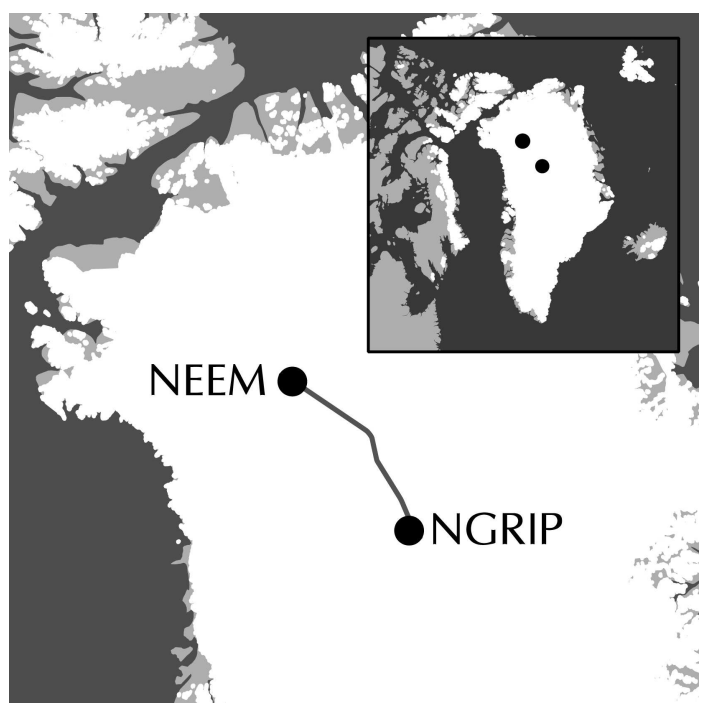

Fig. 1. The $423 \mathrm{~km}$ flight line from NGRIP to NEEM in northern Greenland.

Here we present two new automated methods: (1) a simplified method for inferring the local layer slope, which can be used to create initial estimates of layer positions; and (2) a method for tracking a layer from that initial estimate. An iterative optimization technique, known as an active contour model or snake, is used to fit the layer estimate to the echogram, while preserving smooth layers and minimizing accidental jumps to adjacent layers. The combined process is not completely autonomous, as a human operator is required to pick seed points for the initial layer estimates.

\section{DATA}

For this study a sample dataset was chosen from the Center for Remote Sensing of Ice Sheets (CReSIS, University of Kansas, USA) data archive (Gogineni, 2012), which is also available from the US National Snow and Ice Data Center (Leuschen, 2011). The dataset covers a $423 \mathrm{~km}$ transect along the ice divide in northern Greenland (Fig. 1), from the North Greenland Ice Core Project (NGRIP) ice core $\left(75.01^{\circ} \mathrm{N}, 42.53^{\circ} \mathrm{W}\right.$; NorthGRIP Members, 2004) to the North Greenland Eemian Ice Drilling (NEEM) ice core $\left(77.45^{\circ} \mathrm{N}, 51.06^{\circ} \mathrm{W}\right.$; NEEM Community Members, 2013). It was obtained on 6 May 2011 using the Multichannel Coherent Radar Depth Sounder/Imager (MCoRDS/I; Rodriguez-Morales and others, 2014) on a NASA P-3 Orion aircraft during the 2011 Arctic Operation IceBridge Mission (Koenig and others, 2010). The radar was operated with a chirp signal from 180 to $210 \mathrm{MHz}$. After processing, the average horizontal resolution is $13 \mathrm{~m}$, and the vertical range resolution is $2.8 \mathrm{~m}$, assuming a constant dielectric permittivity of $\epsilon_{r}^{\prime}=3.15$ (Koh, 1992; Kovacs and others, 1995).

A correction for higher wave velocity in the lower-density firn was applied for the two-way travel time to depth conversions using a constant offset at each drill site. Manual bedrock picks were made at both drill sites. The offset was found from the difference between the length of the ice core and the distance to bedrock assuming a constant wave velocity of $c / \sqrt{\epsilon_{r}^{\prime}}$, where $c$ is the velocity of light in air. We found the firn corrections to be $15.8 \mathrm{~m}$ for the NEEM site and $8.7 \mathrm{~m}$ for the NGRIP site, which is comparable to firn corrections used at the Greenland Ice Core Project (GRIP) drill site (Hempel and others, 2000).

The NEEM ice-core depth-age profile has been matched to the NGRIP core profile using match points from electrical conductivity measurements (ECM) (Rasmussen and others, 2013). These depth-depth match points were used as ground truth for evaluating the quality of automatically traced layers. Both depth-age profiles of NGRIP and NEEM were extended to the 2011 surface from the year the cores were drilled (1996 and 2008m respectively) by interpolation and assuming that the average present-day accumulation rate at each core site did not change over the period.

\section{METHOD}

The protocol of tracing layers is divided into four discrete steps: (1) preprocessing of the echogram; (2) estimation of the local layer slope; (3) user-assisted picking of seed points for an initial layer estimate; and (4) tracing the layer using a snake. An implementation of the method in the Python programming language is available at http://www. iceandclimate.nbi.ku.dk/data/.

\section{Step 1: preprocessing of the echogram}

As the ice is attenuating the returned signal with depth, it is convenient to apply a high-pass filter to the echogram to draw out the layer features. To obtain the detrended echogram I, we construct a crude high-pass filter by subtracting the echogram $P$ with a low-pass filtered version of itself. The low-pass filter is constructed as a convolution with a Gaussian kernel with spread $\sigma_{d}$.

$$
I(x, y)=P(x, y)-P(x, y) \cdot \frac{1}{2 \pi \sigma_{d}^{2}} \exp \left(-\frac{x^{2}+y^{2}}{2 \sigma_{d}^{2}}\right)
$$

To remove the effect of fluctuating aircraft altitude, we also align the detrended echogram to the picked ice surface.

\section{Step 2: estimation of the local layer slope}

One common step in the typical processing procedure is the horizontal stacking or incoherent averaging of the echogram. This is done under the assumption that the layers are flat within the stacking length. If the layers are flat, this method would effectively preserve the signal and reduce the noise. On the other hand, if the internal layers are slanted, the stacking will decrease the signal.

Horizontal smoothing of the echogram has the same sensitivity to layer slope as the horizontal stacking (Fig. 2). By comparing the smoothed echogram to the original, areas with flat layer sections can be found by their gain in signalto-noise ratio. If the smoothing is applied at an angle $\theta$, the areas with a layer slope similar to $\theta$ can be detected in the same way. We use this property to infer the slope of the local layers, by applying a number of slanted Gaussian filters, $h(x, y, \sigma, \theta)$.

$$
\begin{aligned}
& h(x, y, \sigma, \theta)= \\
& 12 \pi \sqrt{\sigma_{x}^{2} \sigma_{y}^{2}} \exp \left(-\frac{(x \cos \theta-y \sin \theta)^{2}}{2 \sigma_{x}^{2}}+\frac{(x \sin \theta+y \cos \theta)^{2}}{2 \sigma_{y}^{2}}\right)
\end{aligned}
$$

The filter spread, $\sigma$, is chosen $\sigma_{x} \gg \sigma_{y}$ so the filter will primarily smooth along the local layering (Fig. 2c). The smoothed echogram $I_{\mathrm{s}}$ can be computed as a convolution between the detrended echogram I and the filter, which can 

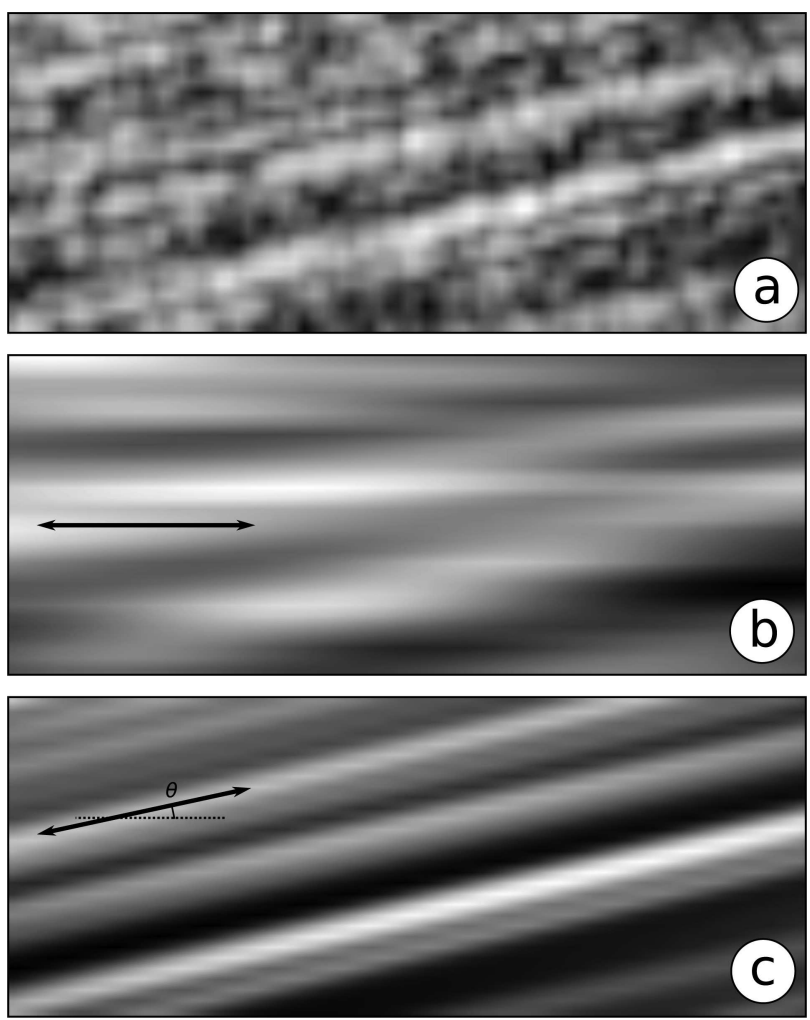

Fig. 2. The effect of slope-aware smoothing. (a) An echogram showing internal sloping layers; (b) smoothing along the horizontal axis; and (c) smoothing along the local layer slope $\theta$. Direction of smoothing is indicated by the arrows, using $\sigma_{x}=15$ and $\sigma_{y}=0.5$.

be done efficiently in the frequency domain. The local layer slope $\Theta$ for each pixel $(x, y)$ can be found as the $\theta \in\left\{-\theta_{\max }, \ldots, \theta_{\max }\right\}$ that yielded the strongest response $I_{\mathrm{r}}$ to the convolution.

$$
\begin{aligned}
I_{\mathrm{s}}(x, y, \theta)_{\sigma} & =I(x, y) \cdot h(x, y, \sigma, \theta) \\
I_{\mathrm{r}}(x, y) & =\max _{\theta} I_{\mathrm{s}}(x, y, \theta)_{\sigma} \\
\Theta(x, y) & =\underset{\theta}{\arg \max } I_{\mathrm{s}}(x, y, \theta)_{\sigma}
\end{aligned}
$$

The areas without layer structure will have a random local layer slope with this method (Fig. 3b), and response to the filter $I_{r}$ will be low. To avoid bands of noise in the echograms where no visible layers are present, we filter the echogram vertically using splines assuming that the layer slope is continuous and smooth. Interpolating the slope estimate $\Theta(x, y)$ using $I_{r}$ as weights for the spline yields a smoothed local layer slope $\Theta_{c}$ which fills in the interpolated slope for regions without layer structure (Fig. 3c). We set the filter response used as weights to $I_{\mathrm{r}}=0$ at the bedrock using a coarse pick in order to increase slope estimate accuracy for the near-bedrock layers. If this were not done, the slope of the bedrock would dominate due to the stronger reflection.

\section{Step 3: seed points to layer estimates}

Sime and others (2011) showed that integrating the local layer slope can provide first-order approximations to the locations of layers. We set up an interactive user interface for an operator to provide initial seed points on a layer to initialize the integration. For each added point, the initial layer estimate is updated by the distance-weighted average of the mutual integration between pairs of points. The
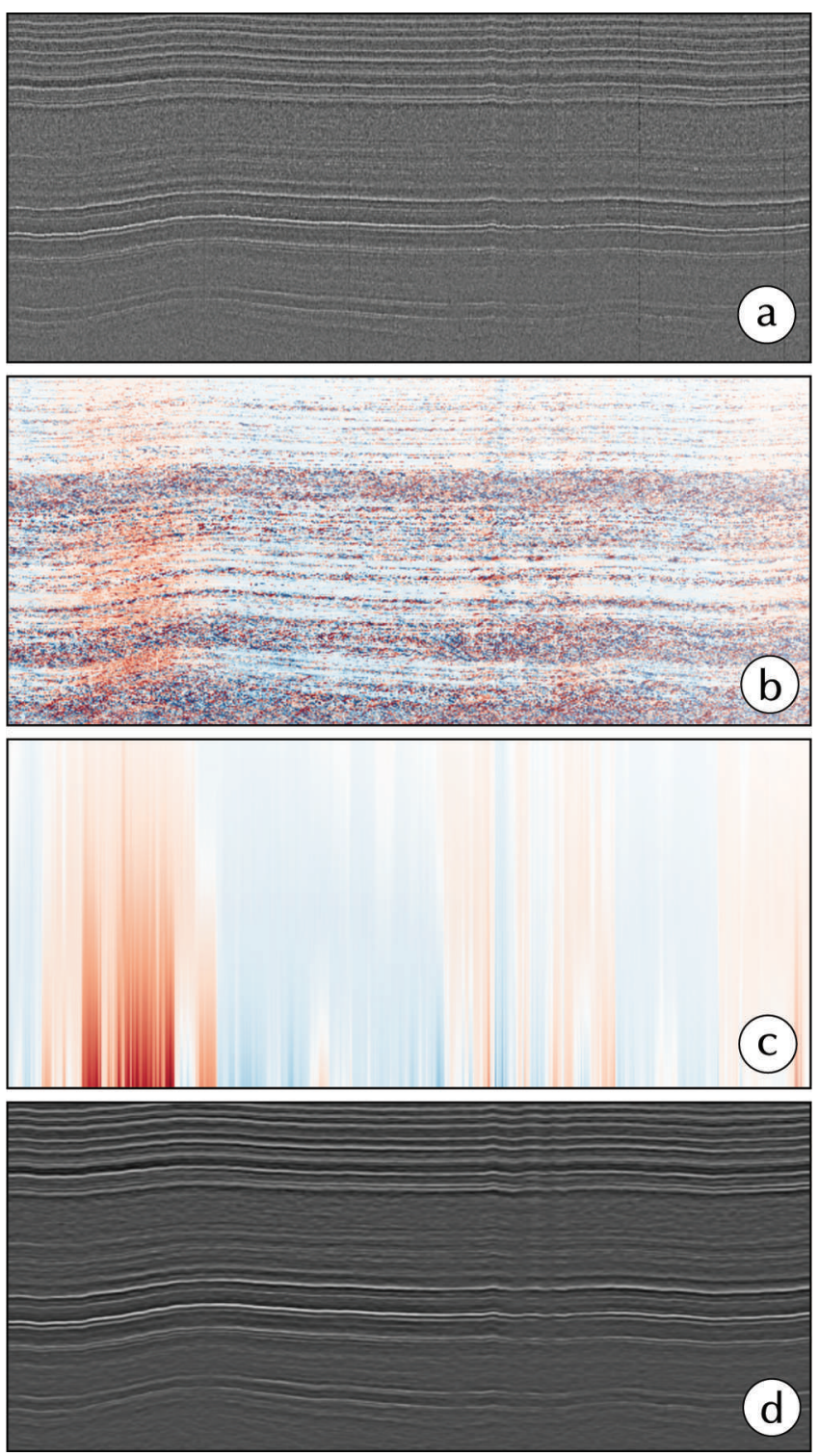

Fig. 3. Estimation of local layer slope. (a) The detrended echogram I; (b) local layer slope $\Theta$, with red areas indicating left-to-right ascending layers and blue indicating descending layers; (c) the cleaned local layer slope $\Theta_{c}$; and (d) the smoothed echogram $I_{\mathrm{s}}\left(x, y, \Theta_{c}\right)$.

number of operator picks needed depends on the quality of the local layer slope, as small errors in the slope estimation quickly propagate.

\section{Step 4: tracing layers with snakes}

An active contour model (Kass and others, 1988) is used to fit the synthetic layer estimates to the echogram. Often referred to as a snake, it consists of a number of knots, interconnected like a string of beads, forming a piecewise linear layer. A snake $v$ with $N$ knots, each described by their location $(x, y)$, can be defined as

$$
v_{i}=[x(i), y(i)], i \in\{1,2, \ldots, N\}
$$

Each knot can move within a predefined neighborhood, and the movement is constrained by an internal and external energy function. The energy functions are chosen to have minimal energy when the snake matches an internal layer in the echogram. 


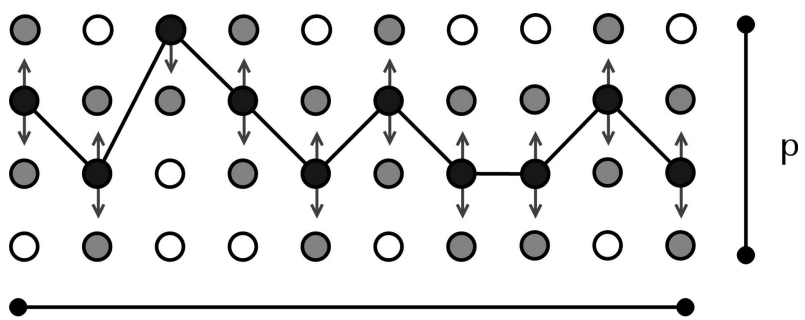

q

Fig. 4. One snake (in black) represented on a $q$-by-p sized trellis where only vertical movement is allowed. The grey nodes represent the neighborhood of possible movement for each knot.

The internal energy only depends on the shape of the layer. In the case of fitting internal layers, a desirable internal energy would preserve smooth continuous layering by minimizing the curvature of the snake. The internal energy function $E_{\text {int }}(v)$ is chosen to penalize sudden changes in layer slope, where $\gamma$ is a scaling parameter and $\phi\left(v_{i}\right)$ is the angle formed between the $v_{i}-v_{i-1}$ and $v_{i+1}-v_{i}$ edges in the snake.

$$
\begin{aligned}
E_{\text {int }}(v) & =\sum_{i=2}^{N-1}\left(\gamma^{\left|\phi\left(v_{i}\right)\right|+1}-\gamma\right) \\
\cos \phi(v(i)) & =\frac{\left(v_{i}-v_{i-1}\right) \cdot\left(v_{i+1}-v_{i}\right)}{\left|v_{i}-v_{i-1}\right|\left|v_{i+1}-v_{i}\right|}
\end{aligned}
$$

The external energy only depends on the configuration of the snake relative to the image, or echogram in this case. To cover cases where a layer might briefly disappear, the external energy is chosen to consist of both the mean intensity of the proposed layer $E_{\text {ext }}^{\prime}$ along the snake, and a pattern match $E_{\text {ext }}^{\prime \prime}$ evaluating how similar the echogram is in the area $\Psi$ around the knots, where $\psi$ is the pixel offset from the knot.

$$
\begin{aligned}
& E_{\text {ext }}^{\prime}(v)=-\sum_{i=2}^{N} 1\left|v_{i}-v_{i-1}\right| \sum_{(x, y)=v_{i-1}}^{v_{i}} I_{\mathrm{s}}\left(x, y, \Theta_{C}\right) \\
& E_{\mathrm{ext}}^{\prime \prime}(v)=\sum_{i=2}^{N} \sum_{\psi}^{\Psi}\left[I_{\mathrm{s}}\left(v_{i}+\psi, \Theta_{C}\right)-I_{\mathrm{s}}\left(v_{i-1}+\psi, \Theta_{C}\right)\right]^{2}
\end{aligned}
$$

Finally, the optimal snake can be found by choosing a $v$ that yields the lowest energy. To balance the energy contributions of each of the terms, $\alpha$ and $\beta$ are used as scaling parameters.

$$
v_{\text {optimal }}=\underset{v}{\arg \min }\left(\alpha \cdot E_{\mathrm{int}}(v)+\beta \cdot E_{\mathrm{ext}}^{\prime}(v)+E_{\mathrm{ext}}^{\prime \prime}(v)\right)
$$

To simplify the computation, we choose a neighborhood in which the knot can only move vertically, so all possible snakes take the form of a trellis (Fig. 4). If a $q$-by- $p$ trellis were to be exhaustively searched for the snake that yielded the lowest energy, the computational complexity would be $\mathcal{O}\left(q^{p}\right)$. This is not feasible to compute. However, with the iterative invocation of the Viterbi dynamic programming algorithm (Forney, 1973; Amini and others, 1990), the computational complexity is reduced to $\mathcal{O}\left(m q^{d}\right)$, where $m \ll p$ is the size of the search neighborhood, and $d=3$ is the maximum number of knots involved in calculating the energy of a knot.

The initial layer estimate is used as the starting configuration of the snake. For each iteration, all permutations of

\begin{tabular}{|c|c|c|}
\hline \multicolumn{3}{|c|}{ Preprocessing (step 1) } \\
\hline$\sigma_{d}$ & Spread of the low-pass filter & 5 \\
\hline \multicolumn{3}{|c|}{ Slope field (step 2) } \\
\hline$\theta_{\max }$ & Maximum filter slant* & $20^{\circ}, 15^{\circ}$ and 3 \\
\hline$\sigma_{x}$ & Spread for filter along layers & 15,45 and 10 \\
\hline$\sigma_{y}$ & Spread for filter across layers & 0.125 \\
\hline$n$ & Steps between $-\theta_{\max }$ and $\theta_{\max }$ & 10 \\
\hline \multicolumn{3}{|c|}{ Tracing the layers (step 4) } \\
\hline \multirow[t]{2}{*}{$N$} & Number of knots & 305 \\
\hline & Horizontal distance between knots & $\sim 1380 \mathrm{~m}$ \\
\hline$m$ & Size of search neighborhood & 3 \\
\hline$\alpha$ & Overall snake smoothness & 10 \\
\hline$\beta$ & Snake fitting to echogram intensities & 50 \\
\hline$\gamma$ & Maximum kink size of the snake & 6 \\
\hline$\Psi_{x}$ & Along-track pattern match from knot & $\pm \sim 650 \mathrm{~m}$ \\
\hline$\Psi_{y}$ & Depth pattern match from knot & $\pm \sim 200 \mathrm{~m}$ \\
\hline
\end{tabular}
the snake position within the neighborhood are evaluated.
Table 1. Parameters used for processing

Description

Value

*In the echogram coordinate system.

The configuration of the snake that yielded the lowest energy is then used as the starting configuration of the next round of the iteration. If the configuration of the snake was not changed as a result of the iteration, then the lowest energy and optimal snake $v_{\text {optimal }}$ has been found.

The scaling parameters $\alpha, \beta$ and $\gamma$ are tuned by hand to the specific radar system. First the iterative snake algorithm is run using $\alpha=0$, and $\beta$ is tuned to balance the contributions of $E_{\text {ext }}^{\prime}$ and $E_{\text {ext }}^{\prime \prime}$. In this mode, all smoothness constraints have been disabled and the snake will fit a layer for a while, then jump to a new layer, and so on. If $\beta$ is too small, the snake will not be placed at the intensity peak of the layer in the echogram. With a too large $\beta$, the snake will be placed at the global highest intensity in the echogram. Once a balance has been found between the $E_{\text {ext }}^{\prime}$ and $E_{\text {ext }}^{\prime \prime}$ contributions, $\alpha$ can be increased to reduce jumps to adjacent layers. However, if $\alpha$ becomes too large, then the snake will be flat. The $\gamma$ parameter can be used to constrain the size of large kinks in the snake.

\section{RESULTS}

We processed the sample NGRIP-NEEM transect using the slope estimation and smoothing algorithm previously described (steps 1 and 2). The processing parameters are listed in Table 1. The parameter $\sigma_{x}$ is used to control the characteristic length in which the local layer can be considered straight, and $\theta_{\max }$ is the maximum layer slope we would expect that layer to have. We tuned these parameters by hand, although $\theta_{\max }$ was chosen to be larger than the steepest layer slope that could be identified from the echogram. The transect was processed using three sets of the parameter pair $\left(\theta_{\max }, \sigma_{x}\right)$, matching from the steepest to the flattest regions of the internal layers.

Ten layers were picked by an operator using an average of 9.8 seed points per layer, with a maximum of 20 for the deepest layer (step 3). The layers were chosen as they were continuous for the entire transect, except for smaller sections of up to $\sim 6 \mathrm{~km}$ where the signal was lost. As seen in Figure 5, integrating the local layer slope to form a synthetic layer provides a useful estimate of the actual location of an 


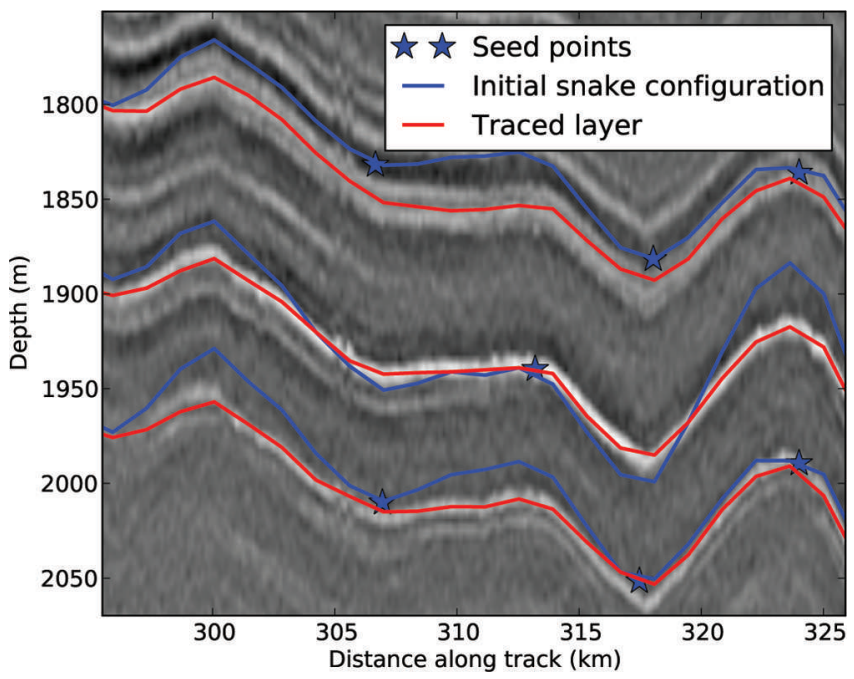

Fig. 5. Tracing layers from seed points. The initial snake configuration (blue lines) from integrating the local layer slope was evolved from the user-picked seed points (blue stars). The layers were traced (red lines) using the initial snake configuration as input to the snake algorithm.

internal layer. The initial layer estimates were then optimized using the snake-based algorithm (step 4), converging, on average, in 19 iterations. The traced layers are shown in Figure 6 . With a coarse spacing of $1.38 \mathrm{~km}$ between the knots in the snake, it was not possible to resolve all of the high-frequency undulations in the traced layer.

To evaluate the accuracy of the method, we compared the traced layers to the ice-core age-depth scales. From Figure 7 it is clear that the traced layers are in fact isochrones, as layers match the ice-core chronology within the resolution of the radar.

On a standard laptop $(2.3 \mathrm{GHz}$ Intel i7 CPU, $16 \mathrm{~GB}$ memory), the preprocessing of the $423 \mathrm{~km}$ transect can be done in $<30 \mathrm{~min}$. The manual picking of a seed point takes, on average, $1 \mathrm{~min} 30 \mathrm{~s}$ per layer. Automatically tracing a layer from the manual picks takes $<30 \mathrm{~s}$. The automatic layer tracing is not yet optimized for speed, but significant

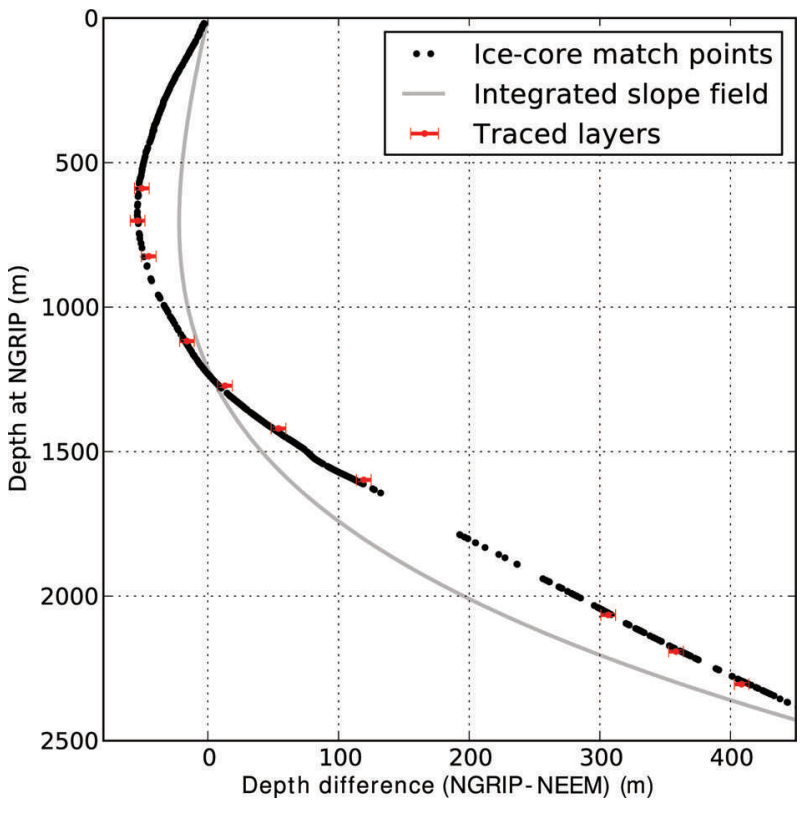

Fig. 7. Comparison of the traced layers with the ice-core dating. The plot shows the depth difference of the features found at each ice-core site, referenced on the NGRIP depth scale. Black points show the isochronous match points in the two ice cores, and red points show the traced layers at the ice-core sites. Error bars for the red traced layers are based on the resolution of the echogram, $\pm 2 \times 2.8 \mathrm{~m}$ (one resolution interval per core intersection). This can be compared to the integration of the slope field from NGRIP to NEEM (grey line), which was done without tracing individual layers.

speed increases can be expected, as the algorithm can easily be parallelized.

\section{DISCUSSION}

In the formulation of the slope-field-generating algorithm (step 3) we assume that the local layer slope is continuous and smooth. For the interior ice sheet we expect this assumption to be valid; however, in some regions with fast

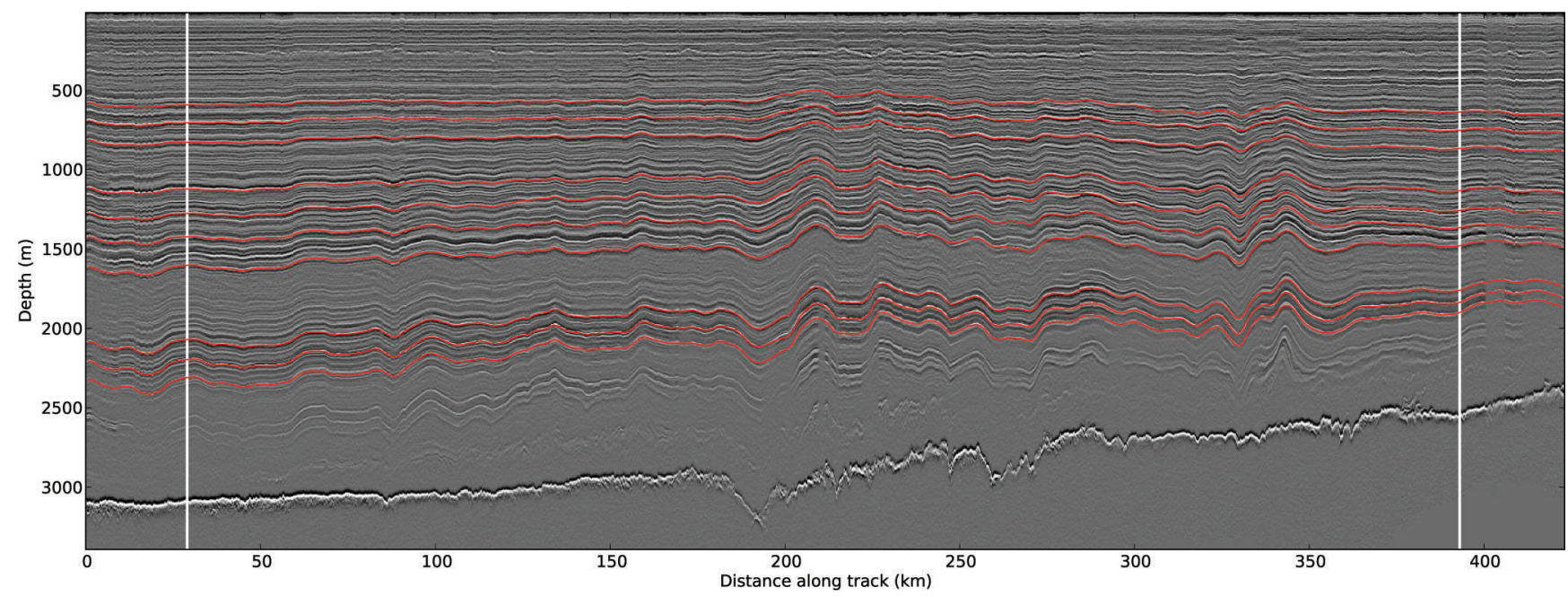

Fig. 6. The full NGRIP-NEEM transect, with the traced layers in red. Ice cores are shown as white vertical lines, with NGRIP to the left and NEEM to the right. The number of picked seed points per layer needed to provide an initial snake configuration averaged 5 for the upper triplet, 9 for the middle four layers and 15 for the lowest triplet. 
ice flow, discontinuities in the visible layers can appear. Using our method to recover slopes in such regions would lead to incorrectly produced slope fields. We do not recommend using our method in areas where the visible stratigraphy is complex, largely missing or near overturning layers, seen near the bedrock in northern Greenland (NEEM Community Members, 2013). Preliminary applications in regions near the margin suggest that the method is robust to large changes in layer slope. The choice of $\theta_{\max }, \sigma_{x}$ and $N$ determines how well layers are traced in such regions. For processing regions with large layer slope, a conservative choice of a large maximum slope angle $\theta_{\max }$ can be determined directly from the echograms, and should be accompanied by reducing $\sigma_{x}$, the characteristic length in which the layer can be seen approximated as straight. An increased number of snake knots $N$ is also needed to provide a better layer trace, as the traced layer is, by the definition of the snake, piecewise linear between the knots.

Changing the tracing method paradigm from propagation along the track, to tracing the entire layer within two end points, raises new operator challenges. When tracing along track, the algorithm or operator has been required to come up with a metric of when to stop due to the layer quality changing. When tracing the entire layer at the same time, the question is, rather, whether the initial layer estimate for the tracing algorithm is of high enough quality. If the estimate is of bad quality (e.g. crosses multiple layers in the echogram), the snake might get stuck in a local minimum and not trace the intended layer. The quality of the layer estimate can be increased by improvements to the slope field algorithm or by adding additional seed points. Optimizing the layer tracing (step 4) by parallelization has the potential to provide real-time results. Such instant feedback would improve the usability of an interactive layer-tracing tool by an iterative application of steps 3 and 4.

The parameter $I_{\mathrm{r}}$ is a measure of how well the filter matches the layers, and we expect that it can be used as an uncertainty estimate of the slope field. The uncertainty of the slope estimate increases in regions without a clear stratigraphy, which was also reflected by the number of seed points needed to provide an initial layer estimate for the deepest triplet of layers (Fig. 6). Incorporating the slope uncertainty into the snake-tracing energy functions or neighborhood selection can further improve the layer trace.

\section{CONCLUSION}

We have developed two new automated methods for tracing internal layers in radio echograms. The two methods can be used in tandem or applied individually to produce layer slope fields or to optimize existing layer traces. When we apply the methods to trace ten layers over a $423 \mathrm{~km}$ transect between two deep ice cores with known age-depth relations, we can verify that the tracing algorithm produces isochrones that match the ice cores within the resolution of the echogram.

The layer-tracing method breaks with the traditional paradigm of propagating layer picks along track by fitting all points of the layer at the same time to the echogram. Automated layer tracing can be done in near-real time from picked seed points, given a preprocessing of the echogram to extract the layer slope. This will allow human operators to be more productive in tracing large-scale datasets.

\section{ACKNOWLEDGEMENTS}

We acknowledge the use of data and data products from CReSIS generated with support from US National Science Foundation grant ANT-0424589 and NASA grant NNX10AT68G. This work was supported by European Research Council Advanced Grant No. 246815 WATERunderthelCE.

\section{REFERENCES}

Amini AA, Weymouth TE and Jain RC (1990) Using dynamic programming for solving variational problems in vision. IEEE Trans. Pattern Anal. Machine Intell., 12(9), 855-866 (doi: 10.1109/34.57681)

Baldwin DJ, Bamber JL, Payne AJ and Layberry RL (2003) Using internal layers from the Greenland ice sheet, identified from radio-echo sounding data, with numerical models. Ann. Glaciol., 37, 325-330 (doi: 10.3189/172756403781815438)

Crandall DJ, Fox GC and Paden JD (2012) Layer-finding in radar echograms using probabilistic graphical models. In Proceedings of the 21st International Conference on Pattern Recognition (ICPR 2012), 11-15 November 2012, Tsukuba, Japan. Institute of Electrical and Electronics Engineers, Piscataway, NJ, 1530-1533

Eisen O, Nixdorf U, Wilhelms F and Miller H (2004) Age estimates of isochronous reflection horizons by combining ice core, survey, and synthetic radar data. J. Geophys. Res., 109(B4), B04106 (doi: 10.1029/2003JB002858)

Fahnestock MA, Abdalati W, Luo S and Gogineni S (2001) Internal layer tracing and age-depth-accumulation relationships for the northern Greenland ice sheet. J. Geophys. Res., 106(D24), 33 789-33 797 (doi: 10.1029/2001JD900200)

Forney GD Jr (1973) The viterbi algorithm. Proc. IEEE, 61(3), 268278 (doi: 10.1109/PROC.1973.9030)

Forster RR and 12 others (2014) Extensive liquid meltwater storage in firn within the Greenland ice sheet. Nature Geosci., 7(2), 9598 (doi: 10.1038/ngeo2043)

Gades AM, Raymond CF, Conway H and Jacobel RW (2000) Bed properties of Siple Dome and adjacent ice streams, West Antarctica, inferred from radio-echo sounding measurements. J. Glaciol., 46(152), 88-94 (doi: 10.3189/172756500781833467)

Gogineni P (2012) CReSIS MCoRDS2 data. Center for Remote Sensing of Ice Sheets, Lawrence, KS. Digital media: http:// data.cresis.ku.edu/

Gudmandsen P (1975) Layer echoes in polar ice sheets. J. Glaciol., 15(73), 95-101

Harrison CH (1971) Radio-echo sounding: focusing effects in wavy strata. Geophys. J. R. Astron. Soc., 24(4), 383-400 (doi: 10.1111/j.1365-246X.1971.tb02185.x)

Hempel L, Thyssen F, Gundestrup N, Clausen HB and Miller H (2000) A comparison of radio-echo sounding data and electrical conductivity of the GRIP ice core. J. Glaciol., 46(154), 369-374 (doi: 10.3189/172756500781833070)

Jacobel RW and Hodge SM (1995) Radar internal layers from the Greenland summit. Geophys. Res. Lett., 22(5), 587-590 (doi: 10.1029/95GL00110)

Karlsson NB, Rippin DM, Vaughan DG and Corr HFJ (2009) The internal layering of Pine Island Glacier, West Antarctica, from airborne radar-sounding data. Ann. Glaciol., 50(51), 141-146

Karlsson NB, Dahl-Jensen D, Gogineni SP and Paden JD (2013) Tracing the depth of the Holocene ice in North Greenland from radio-echo sounding data. Ann. Glaciol., 54(64), 44-50 (doi: 10.3189/2013AoG64A057)

Kass M, Witkin A and Terzopoulos D (1988) Snakes: active contour models. Int. J. Computer Vision, 1(4), 321-331 (doi: 10.1007/ BF00133570)

Koenig L, Martin S, Studinger M and Sonntag J (2010) Polar airborne observations fill gap in satellite data. Eos, 91(38), 333-334 (doi: 10.1029/2010EO380002) 
Koh G (1992) Dielectric constant of ice at 26.5-40 GHz. J. Appl. Phys., 71(10), 5119-5122 (doi: 10.1063/1.350616)

Kovacs A, Gow AJ and Morey RM (1995) The in-situ dielectric constant of polar firn revisited. Cold Reg. Sci. Technol., 23(3), 245-256 (doi: 10.1016/0165-232X(94)00016-Q)

Leuschen C (2011) IceBridge MCoRDS L1B geolocated radar echo strength profile: frames 20110506_01_019, 20110506_01_027. NASA Distributed Active Archive Center/National Snow and Ice Data Center, Boulder, CO. Digital media: http://nsidc.org/data/ irmer1b

Mottram R and 7 others (2009) A new regional high-resolution map of basal and surface topography for the Greenland ice-sheet margin at Paakitsoq, West Greenland. Ann. Glaciol., 50(51), 105-111 (doi: 10.3189/172756409789097577)

NEEM Community Members (2013) Eemian interglacial reconstructed from a Greenland folded ice core. Nature, 493(7433), 489-494 (doi: 10.1038/nature11789)

North Greenland Ice Core Project (NorthGRIP) Members (2004) High-resolution record of Northern Hemisphere climate extending into the last interglacial period. Nature, 431(7005), 147-151 (doi: 10.1038/nature02805)

Rasmussen SO and 23 others (2013) A first chronology for the North Greenland Eemian Ice Drilling (NEEM) ice core. Climate Past, 9(6), 2713-2730 (doi: 10.5194/cp-9-2713-2013)

Robin GdeQ, Evans S and Bailey JT (1969) Interpretation of radio echo sounding in polar ice sheets. Philos. Trans. R. Soc. London, Ser. A, 265(1166), 437-505 (doi: 10.1098/rsta.1969.0063)
Rodriguez-Morales F and 17 others (2014) Advanced multifrequency radar instrumentation for polar research. IEEE Trans. Geosci. Remote Sens., 52(5), 2824-2842 (doi: 10.1109/ TGRS.2013.2266415)

Rotschky G, Eisen O, Wilhelms F, Nixdorf $U$ and Oerter $\mathrm{H}$ (2004) Spatial distribution of surface mass balance on Amundsenisen plateau, Antarctica, derived from ice-penetrating radar studies. Ann. Glaciol., 39, 265-270 (doi: 10.3189/ 172756404781814618)

Siegert MJ (1999) On the origin, nature and uses of Antarctic icesheet radio-echo layering. Progr. Phys. Geogr., 23(2), 159-179 (doi: 10.1177/030913339902300201)

Sime LC, Hindmarsh RCA and Corr HFJ (2011) Automated processing to derive dip angles of englacial radar reflectors in ice sheets. J. Glaciol., 57(202), 260-266 (doi: 10.3189/ 002214311796405870)

Steinhage D, Kipfstuhl S, Nixdorf U and Miller H (2013) Internal structure of the ice sheet between Kohnen station and Dome Fuji, Antarctica, revealed by airborne radio-echo sounding. Ann. Glaciol., 54(64), 163-167 (doi: 10.3189/2013AoG64A113)

Vaughan DG, Corr HFJ, Doake CSM and Waddington ED (1999) Distortion of isochronous layers in ice revealed by groundpenetrating radar. Nature, 398(6725), 323-326 (doi: 10.1038/ 18653)

Welch BC and Jacobel RW (2003) Analysis of deep-penetrating radar surveys of West Antarctica, US-ITASE 2001. Geophys. Res. Lett., 30(8), 1444 (doi: 10.1029/2003GL017210) 